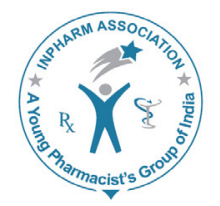

\title{
Detorsion of Twisted (Gangrenous) Ovarian Cyst Followed by Complete Disappearance of the Cyst-A Case Report of Innovative Outcome
}

\author{
Shabbir Ahmad Sheikh ${ }^{1 *}$, Roziana Ramli ${ }^{2}$ and Mainul Haque ${ }^{1}$ \\ ${ }^{1}$ Faculty of Medicine, University Sultan Zainal Abidin, Kampus Kota, Jalan Sultan Mahmud 20400 Kuala \\ Terengganu, Malaysia. \\ ${ }^{2}$ Department of Obstetrics and Gynaecology, Hospital Sultanah Nur Zahirah (HSNZ), Kuala Terengganu, \\ Malaysia.
}

\begin{abstract}
Adnexal torsion in the pediatric and adolescent population is a rare gynecological disease with an incidence of $2.7 \%$. They are categorized by different clinical presentations and by the need to establish a suitable type and timing of treatment in order to prevent problems, such as ovarian necrosis after torsion and infertility. Ovarian torsion denotes a real surgical emergency. Quick diagnosis is crucial for ovarian salvage, and high clinical notion is imperative in this respect. Confounding the diagnosis in overall are more usually come across abdominal complaints in the Emergency Department (ED) such as constipation, diarrhea, and urinary tract infections and more common surgical emergencies such as appendicitis. Until a decade ago,the standard recommended treatment for ovarian torsion was removal of the gangrenous ovary without detorsion. However, conservative management for preservation of ovarian function should be undertaken because most females with adnexal torsion are adolescent girls and of childbearing age. A 10-year-old obese girl presented with a 3-days history of right iliac fossa pain. She was operated for acute appendicitis; however, intraoperatively found gangrenous twisted right ovarian cyst measuring $10 \times 6 \mathrm{~cm}$. Detorsion was done and noted the right fallopian tube appeared to be viable within few minutes however the right ovarian cyst remained the same. The postoperative period was uneventful. The same gangrenous ovary was monitored through repeated ultrasound examinations during follow-up visits and found that cyst was gradually decreasing in size and finally disappeared within 3 months. Detorsion of twisted ovarian cyst (conservative management) leads to complete disappearance and ovary not only be saved but also operative morbidity of interval cystectomy was avoided.
\end{abstract}

Key words: Cyst, Detorsion, Gangrenous, Ovarian, Twisted.

\section{INTRODUCTION}

\begin{tabular}{|c|c|}
\hline \multicolumn{2}{|c|}{ Access this article online } \\
\hline Journal Sponsor & \multirow[b]{2}{*}{$\begin{array}{l}\text { Website: } \\
\text { www.jyoungpharm.org }\end{array}$} \\
\hline \multirow{2}{*}{ www.phcog net } & \\
\hline & $\begin{array}{l}\text { DOI: } \\
\text { 10.5530/jyp.2015.4s.14 }\end{array}$ \\
\hline
\end{tabular}

Adnexal torsion in the pediatric and adolescent population is a rare gynecological disease with an incidence of $2.7 \%{ }^{1}$ Although rare, management of ovarian torsion in children and adolescence could be associated with failure of fertility preservation and endocrine problems. Until a decade ago, the standard recommended treatment for ovarian torsion was removal of the gangrenous ovary without detorsion. ${ }^{2}$

\footnotetext{
*Address for correspondence:

Dr. Shabbir Ahmad Sheikh, Associate Professor and Head of the Unit Obstetrics and Gynecology, Faculty of Medicine, University Sultan Zainal Kampus Kota, Jalan Sultan Mahmud 20400 Kuala Terengganu, Malaysia. E-mail: shabbirahmads@gmail.com
} 


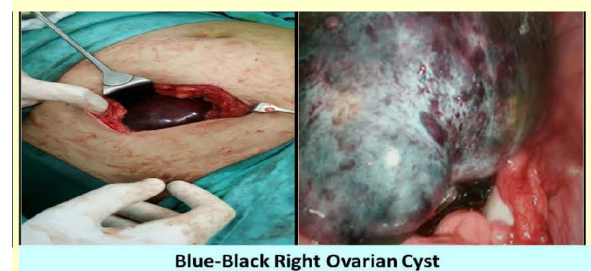

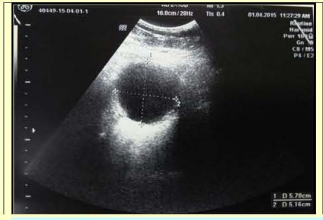

Figure 2a: Few Weeks After Detorsion.

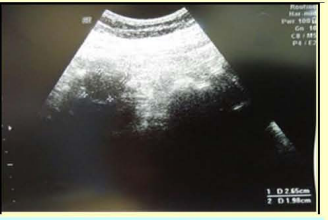

Figure2b: Few Months After Detorsion

Adnexal torsion in the pediatric and adolescent population is a rare gynecological disease with an incidence of $\mathbf{2 . 7 \%}$. They are categorized by different clinical presentations and by the need to establish a suitable type and timing of treatment in order to prevent problems, such as ovarian necrosis after torsion and infertility.

A 10-year-old obese girl presented with a 3-days history of right iliac fossa pain. She was operated for acute appendicitis; however, intraoperatively found gangrenous twisted right ovarian cyst measuring $10 \times 6 \mathrm{~cm}$. Detorsion was done and noted the right fallopian tube appeared to be viable within few minutes however the right ovarian cyst remained the same. The postoperative period was uneventful.

By doing detorsion in this young girl, we not only saved the ovary but also the surgical morbidity.

Graphical Abstract

However, conservative management for preservation of ovarian function should be undertaken because most females with adnexal torsion are adolescent girls and of childbearing age. ${ }^{3-4}$ Detorsion of adnexa helps to preserve ovarian function and maintain fertility. Here, we present a young girl with ovarian torsion who was successfully managed with conservative treatment by detorsion of gangrenous ovarian cyst. She was monitored by repeated ultrasound assessments as out-patient with the gradual disappearance of the cyst within three months after detorsion and we spared not only ovary but also surgical intervention of interval cystectomy.

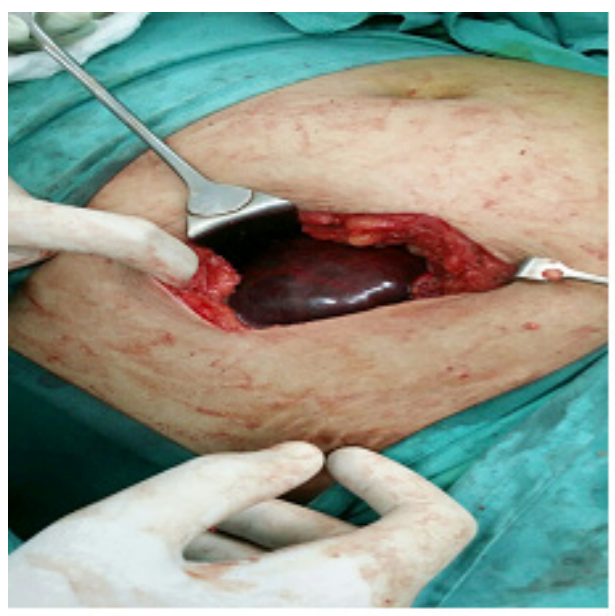

la

\section{CASE REPORT}

A 10-year-old obese premenarchal school girl, a known case of Blount disease, was brought in February 2015 to emergency department, HSNZ, Kuala Terengganu, Malaysia with fever, nausea, vomiting and 3-days history of worsening right iliac fossa pain with pain score of 7-8/10.

On examination, she had tenderness and guarding at right iliac fossa; however, it was difficult to palpate any mass because of pain and the thick abdominal wall. She was febrile with the temperature of $39^{\circ} \mathrm{C}$, pulse rate of $122 \mathrm{bpm}$ and

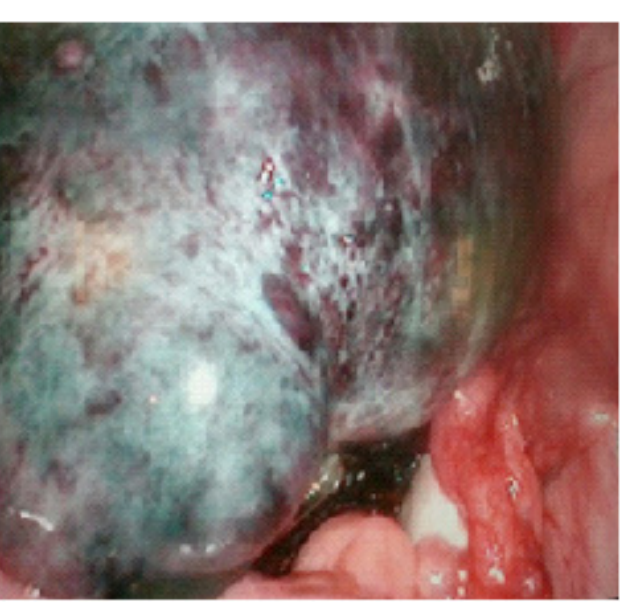

$1 \mathrm{~b}$

Figure 1a and 1b:Blue-Black Right Ovarian Cyst 


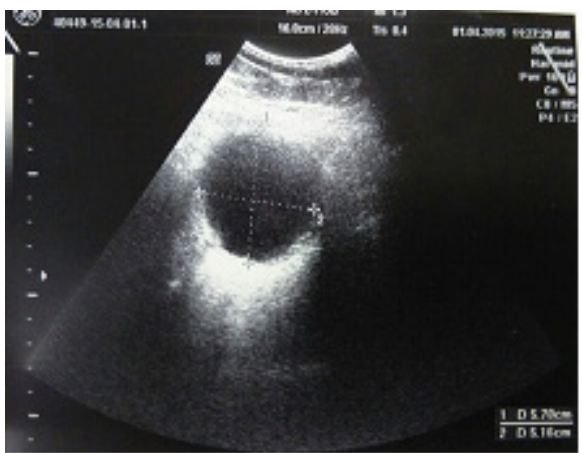

$2 \mathrm{a}$

Figure 2a: Few Weeks After Detorsion

BP of 142/77. Urinalysis was normal and urine pregnancy test was negative. Patient was reviewed by the surgical team and the provisional diagnosis was acute appendicitis.

Abdomen was opened by Lanz incision and intraoperatively the appendix was inflamed and appendectomy was done; however, it was found that there was a right ovarian cyst. The case was referred and reviewed by $O$ and $G$ team on OT-table. Incision was extended a bit for better accessibility to pelvic structures. Uterus and left adnexae appeared normal. However, both the right ovarian cyst and the right fallopian tube were twisted 3-fold around the base and both were blue-black in colour measuring 10x6 cm (Figure 1a and 1b). Adnexal detorsion was performed and shortly after, the twisted fallopian tube appeared viable while the right ovarian cyst remained the same. The postoperative period was uneventful and she was discharged well with the plan of interval cystectomy after 4-6 weeks.

The patient was followed-up every 2 weeks after discharge from hospital. On $1^{\text {st }}$ follow-up visit she was asymptomatic and there was no change in cyst size $(10 \times 6 \mathrm{~cm})$. However, on $2^{\text {nd }}$ follow-up visit when ultrasound was done it was noted that the cyst size had reduced dramatically to $5 \mathrm{x} 5$ $\mathrm{cm}$ (Figure 2a) from intra-operative size and 2-weeks detorsion size of $10 \times 6 \mathrm{~cm}$. Family members were informed that in view of cyst size getting smaller and the patient is asymptomatic, can wait and there is no urgency for interval cystectomy. On $3^{\text {rd }}$ follow-up visit which was three months post-detorsion, ultrasound showed normal size uterus and surprisingly there was no more cyst seen (Figure 2b). Family members were very happy to know this and patient was discharged from Gynae clinic.

\section{DISCUSSION AND CONCLUSION}

Oophorectomy was used to be the standard treatment modality in cases of twisted ischemic (gangrenous)

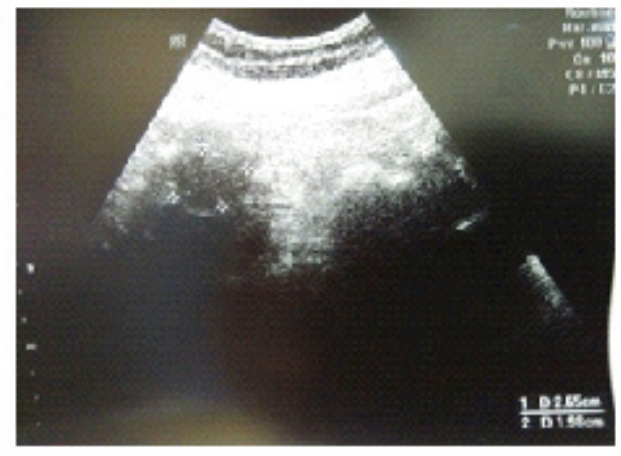

$2 \mathrm{~b}$

Figure 2b: Few Months After Detorsion

ovary until a decade ago ${ }^{5}$ because it was believed that unwinding of the pedicle of the twisted ovary was associated with the risk of thromboembolism based on the assumption that thrombus in the ovarian vein would be released into the systemic circulation. ${ }^{6}$ Secondly, since the ovary is no longer viable, ovarian removal is necessary to prevent infectious morbidity, sepsis and even mortality. Thirdly, fear of malignancy also influences the decision to remove the ovary completely in cases of twisted ovarian cyst. ${ }^{7}$

However, many studies have reported the excellent recovery of ovarian function after conservative approach regardless of the necrotic appearance of the twisted ischemic ovary. ${ }^{1-6,8-12}$ Adnexectomy should be avoided as recovery of normal ovarian size and function, as shown by follicular development is $88 \%$ to $100 \% .{ }^{8}$ Even gangrenous-appearing adnexa should not be removed because it is impossible to predict the chances of the ovary reviving after detorsion. ${ }^{3}$ Secondly, the perceived risks of infectious morbidity, sepsis and mortality have been over estimated. Malignant ovarian neoplasms account for only $1 \%$ of all childhood malignancies. ${ }^{13}$ One literature review found no cases of pulmonary embolism occurring from a thrombosed vein. ${ }^{14}$ Furthermore, it is reported that adnexal torsion patients underwent detorsion without oophorectomy and there were no patient $(n=102)$ in this study had clinical signs of thromboembolism. ${ }^{15}$

Ovarian cystectomy is the surgery of choice for presumed benign masses, however, in torsion cases; there is no clear plane of separation between the cyst and its bed. Excision of a cyst may cause an undue amount of ovarian tissue to be removed inadvertently so detorsion is preferred over oophorectomy. ${ }^{15}$ Somemore, necrotic-appearing ovaries demonstrate follicular activity on sonography weeks after conservative management. ${ }^{5,13}$ Furthermore, the rate of malignancy in paediatric cases of ovarian torsion is low $(1 \%) .{ }^{14}$ 
This case illustrates the successful and innovative outcome of conservative surgery (detorsion) in dealing with ovarian torsion with complete disappearance of ovarian cyst sparing not only ovary but also operative morbidity of interval cystectomy.

\section{ACKNOWLEDGEMENT}

Authors are much grateful to the patient and her family.

\section{CONFLICT OF INTEREST}

This study obtain no funding. Authors possess no conflict of interest.

\section{ABBREVIATION}

\author{
ED: $\quad$ Emergency Department \\ HSNZ: Hospital Sultanah Nur Zahirah
}

\section{Highlights of Paper}

- Adnexal torsion in the pediatric and adolescent population is a rare gynecological disease with an incidence of $2.7 \%$.

- Until a decade ago, the standard recommended treatment for ovarian torsion was removal of the gangrenous ovary without detorsion.

- A 10-year-old obese girl presented with a 3-days history of right iliac fossa pain. She was operated for acute appendicitis; however, intraoperatively found gangrenous twisted right ovarian cyst measuring $10 \times 6 \mathrm{~cm}$.

- Conservative management of twisted ovarian cyst leads to complete disappearance and ovary not only be saved but also operative morbidity of interval cystectomy was avoided.

\section{Author Profile}

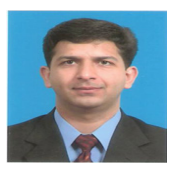

- Dr Shabbir Ahmad Sheikh: Is a Associate Professor and Head of the Unit of O and G, Universiti Sultan Zainal Abidin, Malaysia. He is a medical doctor with specialisation in Obstetrics and Gynecology. He has special interest in Gynecological Oncology; and also have tremendous interest in teaching undergraduate medical students and research in medical education.

\section{REFERENCES}

1. Pansky M, Abargil A, Dreazen E, Golan A, Bukovsky I, Herman A. Conservative management of adnexal torsion in premenarchal girls. J Am Assoc Gynecol Laparosc. 2000; 7(1): 121-4.

2. Tandulwadkar S, Shah A, Agarwal B. Detorsion and conservative therapy for twisted adnexa: our experience. J Gynecol Endosc Surg. 2009; 1(1): 21-6.

3. Silvana V, Hadi K. Detorsion of ovarian cyst: a case report of conservative management. Department of Obstetrics and Gynaecology, Faculty of Medicine University of Indonesia, Indonesia. http://www.bcgip.com/China/Uploads/Editor/PDF/Silvana_2.pdf

4. Yiu-Tai Li, Lung-ChingKuon, Po-Ning Lee, Tsung-Cheng Kuo. Laparoscopic Detorsion of Twisted Ovary. Journal of the Chinese Medical Association 2005; 68(12): 595-8.

5. Aziz J, Davis V, Allen L, Langer JC. Ovarian torsion in children: is oophorectomy necessary? J Pediatr Surg. 2004; 39(5): 750-3.

6. Rody A, Jackisch C, Klockenbusch W, Heinig J, Coenen-Worch V, Schneider HP. The conservative management of adnexal torsion-a case-report and review of the literature. Eur J Obstet Gynecol Reprod Biol. 2002; 101(1): 83-6.

7. Skinner MA, Schlatter MG, Heifetz SA, Grosfeld JL. Ovarian neoplasms In children. Arch Surg. 1993; 128(8): 849-53.

8. Oelsner G, Shashar D. Adnexal torsion. Clin Obstet Gynecol. 2006; 49(3): 459-63.
9. Ahmet Çelik, Orkan Ergün, Hakan Aldemir, Coşkun Özcan, Geylani Özok, Ata Erdener, et al. Long-term results of conservative management of adnexal torsion in children. Journal of Pediatric Surgery 2005; 40(4): 704-8.

10. Cohen SB, Oelsner G, Seidman DS, Admon D, Mashiach S, Goldenberg M. Laparoscopic detorsion allows sparing of the twisted ischemic adnexa. J Am Assoc Gynecol Laparosc. 1999; 6(2): 139-43.

11. Kirkham YA, Lacy JA, Kives S, Allen L. Characteristics and management of adnexal masses in a Canadian pediatric and adolescent population J Obstet Gynaecol Can. 2011; 33(9): 935-43.

12. Breech L, Hillard P. Adnexal torsion in pediatric and adolescent girls. Curr Opin Obstet Gynecol. 2005; 17(5): 483-9.

13. Grovas A, Fremgen A, Rauck A, Ruymann FB, Hutchinson CL, Winchester DP, et al. The National Cancer Data Base report on patterns of childhood cancers in the United States. Cancer 1997; 80(12): 2321-32.

14. Wagaman R, Williams RS. Conservative therapy for adnexal torsion: case report. J Reprod Med. 1990; 35(8): 833-4.

15. Oelsner G, Cohen SB, Soriano D, Admon D, Mashiach S, et al. Minimal surgery for the twisted schaemic adnexa can preserve ovarian function. Hum Reprod. 2003; 18(12): 2599-2. 